\title{
Quelques problèmes actuels d'hydraulique agricole étudiés au cours de deux récentes réunions internationales
}

\author{
(TEXTE RÉSUMÉ dE LA COMMUNICATION PRÉSENTÉE EN SÉANCE)
}

\section{Some problems in contemporary agricultural hydraulics studied at two recent international conferences}

\author{
PAR M. CARLIER, \\ INGÉNIEUR EN CHEF DU GÉNIE RURAL, \\ SEcrétatre GÉNÉral de La COMMISSION INTERNationale \\ DU GÉNIE RURAL
}

\begin{abstract}
Exposé synthétique de l'état actuel des recherches poursuivies depnis plusieurs années, sur le plan international, dans le domaine de l'Hydraulique agricole, telles qu'elles résultent de la réunion régionale de la Commission Internationale des Irrigations et du Drainage $\dot{a}$ Utrecht (septembre 1962) et des journees d'études de la première Section technique de la Commission Internationale du Génie rural, en Avignon (octobre 1962). Situation actuelle comparative des recherches poursuivies aux PaysBas et en France dans le domaine da drainage par canalisations souterraines.
\end{abstract}

\begin{abstract}
A brief summary is given of the present state of agricultural hydraulics research carried out during the last few years on an international scale, as seen from the regional conference of the International Commission on Irrigation and Drainage at Utrecht (September 1962) and the session of the first technical section of the International Commission of Agricultural Engineering at Avignon (October 1962). A comparison is given between the present state of research in France and the Netherlands in drainage by underground duct systems.
\end{abstract}

Au cours des mois de septembre et octobre derniers, se sont tenues deux importanles réunions internationales consacrées à l'étude de problèmes scientifiques et techniques ressortissant à l'hydraulique agricole.

1. Du 25 au 28 septembre, le Comité national néerlandais de la Commission internationale des irrigations et du drainage a organisé, à Utrecht, des séances de travail qui ont rassemblé 171 par- ticipants représentant 24 pays; les séances d'études ont porté sur les deux thèmes suivants :

- aménagement des eaux dans les zones de deltas et d'estuaires,

- aménagement des eaux dans les polders.

Les deux journées de travail à Utrecht ont été complétées par deux journées de visites techniques, l'une consacrée aux travaux de réalisation du «plan Delta», l'autre à l'aménagement des 
polders du lac d'IJssel (ancien golfe du Zuyderzee).

2. Du 2 au 5 octobre, la $1^{\text {re }}$ Section technique de la Commission internationale du Génie rural a tenu, en Avignon, des séances de travail qui ont groupé 135 participants représentant 13 pays; deux fonctionnaires de la division de la mise en valeur des terres et des eaux de la F.A.O. assistaient également aux réunions.

Les sujets suivants ont été étudiés (1) :

- Comparaison des anciens et nouveaux systèmes d'irrigation collective et leur organisation technique;

- Nouvelles méthodes de prospection et de captage des eaux souterraines pour l'irrigation;

- Evaluation du déficit en eau des plantes cultivées en fonction du climat;

- Aspect physiologique du drainage : détermination de la cote de rabattement de la nappe phréatique;

- Normes de drainage en fonction du milieu physique.

Les deux journées de travail à Avignon ont été complétées par deux journées de visites techniques, l'une sur les installations et les chantiers de la Compagnie nationale d'aménagement de la région du Bas-Rhône et du Languedoc, l'autre à la Société du Canal de Provence et d'aménagement de la région provençale et à l'échelon du Tholonet du Centre de Recherches et d'Expérimentation de Génie rural.

Nous nous proposons de résumer ici les idées essentielles qui se sont dégagées de la présentation et de la discussion des diverses communications présentées à ces deux groupes de réunions, en insistant sur celles qui nous paraissent devoir intéresser le Comité technique de la S.H.F.

Nous grouperons l'ensemble des questions étudiées en distinguant :

- d'une part celles concernant l'irrigation;

- d'autre part celles concernant l'assainissement et, plus spécialement, le drainage à l'aide de canalisations souterraines.

La coexistence, dans les régions méridionales de la métropole, de réseaux d'irrigation anciens et modernes a provoqué la présentation, aux journées d'études d'Avignon, de rapports dégageant très clairement les données comparatives entre ces deux systèmes.

Rappelons, au préalable, que les systèmes d'irrigation traditionnelle qui équipent les anciens réseaux tels que ceux de Carpentras et du vieux Verdon utilisent généralement l'irrigation par ruissellement, l'eau étant vendue à la surface ou au litre/seconde continu, la distribution étant réalisée suivant le système $d u$ «tour d'eau » ou de l'horaire d'arrosage.

Les réseaux modernes, tels ceux du BasRhône-Languedoc, du canal de Provence ou des extensions du canal du Verdon, utilisent l'aspersion; l'eau est vendue au volume effectivement consommé et la distribution est réalisée à la demande.

Les comparaisons des anciens et nouveaux systèmes d'irrigation ont confirmé les avantages bien connus des réseaux modernes sur le plan de l'utilisation rationnelle de l'eau, en particulier l'économie de consommation, mais l'accent a été mis également sur le développement plus rapide des zones irriguées et la réduction des dépenses d'exploitation sur les nouveaux périmètres.

Les journées d'études d'Avignon ont fourni l'occasion à M. Tunc, Maître de recherches au Laboratoire des sols du Centre National de Recherches Agronomiques de Versailles, de présenter les nouvelles formules qu'il a mises récemment au point pour l'évaluation de l'évapo-transpiration potentielle décadaire ou mensuelle d'un périmètre irrigué.

Ces formules ont le mérite d'être très simples et ne nécessitent que la connaissance de grandeurs météorologiques faciles à mesurer; leur utilisation est encore facilitée par l'emploi d'abaques dressés par le Service technique central des travaux d'Hydraulique du Ministère de l'Agriculture.

C'est surtout le domaine du drainage par canalisations souterraines qui a donné lieu à la présentation de communications fort intéressantes dont les conclusions rapportées, tant à Utrecht qu'en Avignon, se recoupent et se confirment mutuellement sur un certain nombre de points.

Dans l'hypothèse d'un régime permanent, les formules de l'Ecole néerlandaise de Hooghoudt et Ernst semblent marquer le terme des études poursuivies depuis près de vingt-cinq ans, à partir de l'ancienne formule de l'ellipse de Blanc, Porchet et Kostiakov, pour mieux traduire l'influence de la profondeur du substratum imperméable, l'incidence de la composante verticale des vitesses, ainsi que la présence de plusieurs couches de sols de perméabilités différentes.

La prise en compte de la vitesse d'abaissement de la surface libre de la nappe, c'est-à-dire l'étude des nappes de drainage en régime variable, fait actuellement l'objet des préoccupations des chercheurs. Cette notion est étroitement liée

(1) Le compte rendu complet des journees d'études d'Avignon comportant le texte des communications a eté édité par M. le Professeur Ragamey, Président de la 1re Section de la C.I.G.R. - Cité Devant, 14, Lausanne (Suisse). 
à l'aspect économique du problème, puisqu'un abaissement plus rapide du front de saturation nécessitera un réseau de canalisations plus serré, donc une augmentation des dépenses d'investissement, mais autorisera une augmentation plus importante du revenu brut des terrains drainés et inversement.

La solution la meilleure sur le plan économique, e'est-à-dire celle qui provoquera l'augmentation maximale du revenu net, pourra donc différer de la solution techniquement parfaite.

Les réunions d'Utrecht et d'Avignon ont permis - précisément - de comparer les premiers résultats obtenus par l'école hollandaise à l'issue des recherches thériques et expérimentales poursuivies à l'occasion du drainage des polders du lac d'IJssel et par l'école française qui, sous l'impulsion de M. Guvon, Ingénieur du Génie rural au Centre de Recherches et d'Expérimentation de Génie rural, a entrepris, depuis deux ans, des études théoriques appuyées sur des observations recueillies sur un réseau expérimental de drainage dans la région parisienne.

Ces deux séries de recherches se recoupent d'ailleurs, pour l'essentiel de leurs résultats.
Dans le cas où le substratum imperméable est en dessous des files de drains, M. GuYoN propose d'assimiler la forme de la directrice de l'ellipse qui, dans le cas où les files de dráins reposent sur le substratum imperméable, recoupe bien les résultats expérimentaux et peut servir de base à des analyses plus poussées pour traduire l'écoulement en régime variable.

Un des points les plus remarquables qui se dégagent des premiers résultats obtenus par M. Guyon est l'intérêt de l'ancienne formule de la surface libre de la nappe à une parabole; ce point doit faire toutefois l'objet de vérifications expérimentales qui viennent d'être entreprises.

Ces deux groupes de résultats présentent lavantage de conduire à des formules du régime variable, beaucoup plus simples que celles déduites de la théorie de base de Boussinesq.

Enfin, les réunions d'Avignon ont également fourni l'occasion de confronter ces études de caractère mathématique à celles, de caractère plutôt qualitatif, qui se développent, parallèlement, au sein d'un corps de doctrine tendant à faire appel aux caractéristiques pédologiques du terrain considéré, pour en déduire les caractéristiques du réseau de drainage convenable.

\section{COMMENTAIRE}

\section{Président M. BEcken}

M. le President remercie vivement M. CArLier de sa communication très synthétique, mais très dense et très riche de substance, et magnifiquement illustrée par des liapositives.

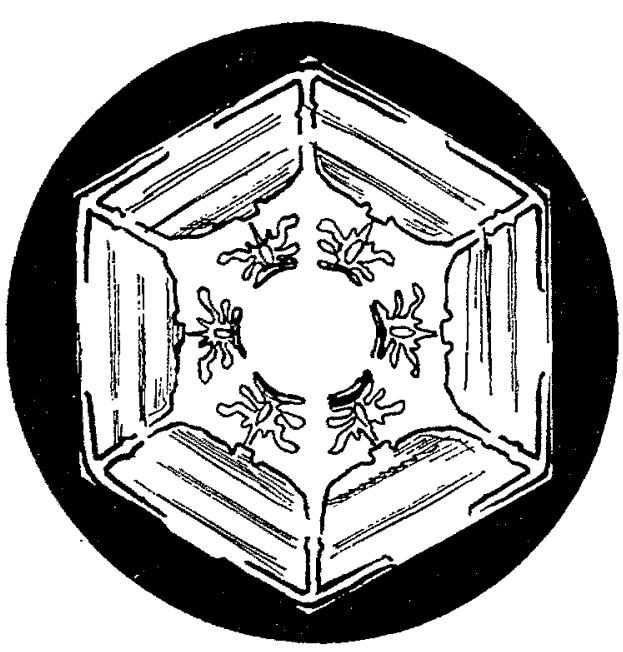

